\title{
Lidil
}

Revue de linguistique et de didactique des langues

$59 \mid 2019$

Prononcer les langues : variations, émotions, médiations

\section{Michèle Monte, Stéphanie Thonnerieux et Philippe Wahl (dir.), Stylistique \& méthode. Quels paliers de pertinence textuelle?}

Lyon, Presses universitaires de Lyon, collection « Textes \& Langue », 2018, $371 \mathrm{p}$.

\section{Laetitia Gonon}

\section{OpenEdition}

\section{Journals}

Édition électronique

URL : http://journals.openedition.org/lidil/6009

DOI : 10.4000/lidil.6009

ISSN : $1960-6052$

\section{Éditeur}

UGA Éditions/Université Grenoble Alpes

Édition imprimée

ISBN : 978-2-37747-090-7

ISSN : $1146-6480$

\section{Référence électronique}

Laetitia Gonon, « Michèle Monte, Stéphanie Thonnerieux et Philippe Wahl (dir.), Stylistique \& méthode. Quels paliers de pertinence textuelle? ", Lidil [En ligne], 59 | 2019, mis en ligne le 01 mai 2019, consulté le 24 septembre 2020. URL : http://journals.openedition.org/lidil/6009; DOI : https://doi.org/10.4000/ lidil.6009

Ce document a été généré automatiquement le 24 septembre 2020.

(C) Lidil 


\title{
Michèle Monte, Stéphanie
} Thonnerieux et Philippe Wahl (dir.), Stylistique \& méthode. Quels paliers de pertinence textuelle?

\author{
Lyon, Presses universitaires de Lyon, collection « Textes \& Langue », \\ 2018, $371 \mathrm{p}$.
}

Laetitia Gonon

\section{RÉFÉRENCE}

Michèle Monte, Stéphanie Thonnerieux et Philippe Wahl (dir.), Stylistique \& méthode. Quels paliers de pertinence textuelle?, Lyon, Presses universitaires de Lyon, collection «Textes \& Langue », 2018, 371 p.

1 Le collectif Stylistique \& méthode paru en 2018 rassemble les Actes d'un colloque de 2015 sur le sujet des unités et des paliers pertinents pour l'analyse stylistique. Le colloque comme l'ouvrage s'inscrivent dans les activités de l'Association internationale de stylistique, créée en 2004, regroupant de nombreux chercheurs et à ce titre représentative de la stylistique comme champ scientifique et institutionnel. Les trois co-directeurs de cette parution sont enseignants-chercheurs en France, à Toulon et Lyon 2.

Ce collectif propose un retour sur la méthodologie de la discipline académique enseignée sous le nom de stylistique: B. Moricheau-Airaud analyse par exemple la méthode préconisée pour le commentaire stylistique aux concours de l'Éducation nationale. La stylistique, qui emprunte ses outils à la linguistique pour les appliquer à la littérature et aux discours, se situe de fait à la croisée de plusieurs disciplines, et se nourrit de leurs apports scientifiques: sémiotique, sémantique interprétative, linguistique de corpus, métrique, analyse de discours, rhétorique, etc. Plusieurs articles 
analysent des extraits de textes littéraires canoniques: par exemple des descriptions des barricades des Misérables de Victor Hugo, qu'étudie U. Yocaris. Cependant les corpus sont divers, et les textes étudiés ne sont pas tous littéraires: les discours politiques (contemporains dans l'article de E. Park-Derrington, prononcés par Lamartine dans l'article de B. Vouillon \& B. Verine), la chanson (S. Chaudier \& J. July) font partie des genres de discours commentés. La dimension épistémologique de l'ouvrage invite les chercheurs à se positionner dans leur champ disciplinaire, et la diversité des approches, qui répond à la diversité des corpus, donne une idée de ce qu'est aujourd'hui le travail en stylistique: une manière féconde d'interpréter les œuvres, littéraires ou non, et de déterminer ce qui fait leur style, ainsi qu'un examen précis des genres de discours non littéraires (F. Revaz étudie le découpage des épisodes dans des bandes dessinées sérielles) et des unités qui composent les textes.

Les méthodes sont parfois contrastées : on peut citer dans ce sens le grand écart entre le travail de P. Jousset et celui de D. Legallois au sein de la même partie. Mais cet ouvrage a le mérite de faire coexister des manières différentes de penser l'interprétation stylistique des textes. Si la démarche corpus driven de D. Legallois repose sur les statistiques obtenues grâce à la détection automatisée de séquences d'unités dans 60 romans du XIX ${ }^{\mathrm{e}}$ siècle, P. Jousset préfère questionner le terme même de méthode : à l'objectivité statistique, il oppose la subjectivité de la lecture interprétative, le parcours. Faut-il être styliste pour être stylisticien? La question est posée par P. Jousset, qui développe une analogie entre les points d'accroche de l'escalade et l'interprétation d'un texte. S. Chaudier et J. July semblent y répondre en acte puisque le style de leur article se veut clairement littéraire. Les différentes parties du collectif se construisent autour de notions méthodologiques (l'articulation local/global, l'énonciation, les sémiologies textuelles...) qui dressent un panorama de la recherche contemporaine en stylistique, mais pensent aussi la stylistique contemporaine en diachronie, comme l'aboutissement d'une réflexion antérieure. Ainsi, certains chercheurs confirmés poursuivent des réflexions menées depuis des années : $\mathrm{F}$. Rastier sur l'unité comme passage; M. Faransis sur trois plans d'énonciation; en initient d'autres comme le fait J.-M. Adam sur le paragraphe (en amont de son ouvrage récemment paru et recensé dans ce même numéro de la revue Lidil). D'autres prolongent et complètent la pensée d'illustres prédécesseurs comme P. Wahl qui reprend le travail de Spitzer sur Céline. D'autres enfin se fondent sur des ouvrages de référence dans leur champ afin de discuter leur pertinence respective : c'est ce que fait S. Thonnerieux sur la strophe en vers libres.

4 La réflexion méthodologique de l'ouvrage porte plus précisément sur les paliers de pertinence pour l'étude en stylistique : comment délimiter les énoncés à étudier ? comment articuler les faits de style ponctuels à une analyse plus globale du texte? Les chercheurs s'accordent sur la nécessité pour le stylisticien de contextualiser historiquement la langue littéraire dans son rapport aux usages discursifs contemporains, afin de ne pas confondre faits de langue et faits de style (C. Lignereux); mais aussi sur la nécessité de contextualiser spatialement un fait de style, en le rapportant à son microcontexte aussi bien qu'au plan mésotextuel - à des unités intermédiaires comme le paragraphe, l'alinéa, le chapitre - et macrotextuel.

On notera la grande richesse de l'ouvrage pour ce qui concerne la réflexion autour des formes poétiques. Une place importante est ainsi donnée à la métrique (É. Delente sur Verlaine), aux unités poétiques (l'unité mésotextuelle de la strophe et sa productivité 
pour l'analyse stylistique chez S. Thonnerieux, S. Chaudier \& J. July), aux figures (les métaphores dans la poésie contemporaine chez Monte). En outre, La poésie est envisagée dans sa matérialité, sa spatialité (S. Orlandi) ou son rapport à la voix dans la partie V.

6 C'est donc le caractère emblématique de cet ouvrage qu'on pourra retenir : il fait état de l'avancement des recherches en stylistique, aussi bien pour ce qui concerne les objets et les outils que les méthodes. Les réalités de la discipline sont contrastées : les analyses passant aisément du microcontexte à la structure de l'œuvre, se font écho. L'ouvrage rend compte d'une diversité qui semble finalement inciter à la collaboration entre stylisticiens aux méthodes moins contradictoires que complémentaires.

\section{AUTEURS}

\section{LAETITIA GONON}

LITT\&ARTS, Université Grenoble Alpes 